\title{
Individual Differences in Teleporting through Virtual Environments
}

Preprint · December 2020

DOI: $10.31234 /$ osf.io/b6cyd

CITATIONS

6

5 authors, including:

2

Lucia A Cherep

The University of Arizona

23 PUBLICATIONS 237 CITATIONS

SEE PROFILE

Anthony James Miller

lowa State University

6 PUBLICATIONS 18 CITATIONS

SEE PROFILE

Some of the authors of this publication are also working on these related projects:

Project $\quad A B M \&$ Regional Food Supply Chains View project

Project path integration View project
READS

119

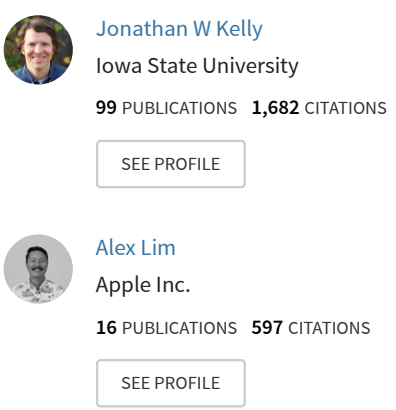




\title{
Individual Differences in Teleporting through Virtual Environments
}

Lucia A. Cherep, Jonathan W. Kelly, Anthony Miller, Alex F. Lim, \& Stephen B. Gilbert

Iowa State University

This manuscript has been accepted for publication and can be found here: https://psycnet.apa.org/ doi/10.1037/xap0000396

\begin{abstract}
Author Note
Pre-registration as well as videos, data, supplementary analyses, supplementary figures, and experiment code are available on the Open Science Framework (doi:10.17605/OSF.IO/XC8V5). This material is based upon work supported by the National Science Foundation under Grant No. CHS-1816029. Preliminary results are described in Cherep, Lim, Kelly, Miller, and Gilbert (2020).

Correspondence concerning this article should be addressed to Lucia Cherep, Department of Psychology, Iowa State University, Ames, IA, 50011-1041. Contact: lacherep@iastate.edu
\end{abstract}




\begin{abstract}
Virtual reality (VR) allows users to walk to explore the virtual environment (VE), but this capability is constrained by real obstacles. Teleporting interfaces overcome this constraint by allowing users to select a position, and sometimes orientation, in the VE before being instantly transported without self-motion cues. This study investigated whether individual differences in navigation performance when teleporting correspond to characteristics of the individual, including spatial ability. Participants performed triangle completion (traverse two outbound path legs, then point to the path origin) within VEs differing in visual landmarks. Locomotion was accomplished using three interfaces: walking, partially concordant teleporting (teleport to change position, rotate the body to change orientation), and discordant teleporting (teleport to change position and orientation). A latent profile analysis identified three profiles of individuals: those who performed well overall and improved with landmarks, those who performed poorly without landmarks but improved when available, and those who performed poorly even with landmarks. Characteristics of individuals differed across profiles, including gender, self-reported spatial ability, mental rotation, and perspective-taking; but only perspective-taking significantly distinguished all three profiles. This work elucidates spatial cognitive correlates of navigation and provides a framework for identifying susceptibility to disorientation in VR.
\end{abstract}

Public Significance Statement: Previous work has shown that teleportation causes disorientation. Yet, individuals vary widely in the extent to which they become disoriented, and our study identified the spatial cognitive abilities that underlie these individual differences. Three distinct groups emerged which highlight the individual differences in navigational accuracy as well as integration of available cues during a triangle completion task. Importantly, groups differed on measures of spatial cognitive ability and video game experience. 
Keywords: spatial cognition, individual differences, teleporting, virtual reality, navigation 


\section{Individual Differences in Teleporting through Virtual Environments}

Modern virtual reality (VR) systems allow the user to walk and turn to explore the virtual environment (VE). However, the ability to walk through the VE is limited by real obstacles, such as walls and furniture. Therefore, exploration of all but the smallest VEs requires a locomotion interface that separates movement through the VE from movement of the user's body. The most popular locomotion interface is teleportation (Boletsis, 2017). To teleport, the user positions a marker within the VE and is then instantly transported to the selected location typically without accompanying visual or body-based self-motion cues. The popularity of the teleporting interface is most likely due to its ease of use (Bozgeyikli et al., 2016; Langbehn et al., 2018) and reduced cybersickness compared to interfaces that include smooth visual motion without movement of the body (Rahimi Moghadam et al., 2018).

Despite the many benefits of teleportation, discordance between movement of the body and movement through the VE comes at a spatial cognitive cost. In particular, spatial updating the process of updating self-location during travel - is disrupted in the absence of self-motion cues. For example, in a triangle completion task in which the participant travels two outbound path legs before pointing back to the path origin, performance suffers when translation (change in position) is accomplished by teleportation compared to walking and suffers further when rotation (change in orientation) is accomplished by teleportation compared to real body rotation (Cherep, Lim, Kelly, Acharya, et al., 2020; Kelly et al., 2020). These findings are consistent with prior research indicating the importance of walking (Lhuillier et al., 2020), translational (Ruddle \& Lessels, 2006), and rotational (Klatzky et al., 1998) self-motion cues to spatial updating. The goal of the current project was to characterize individual differences in spatial updating performance when teleporting. 


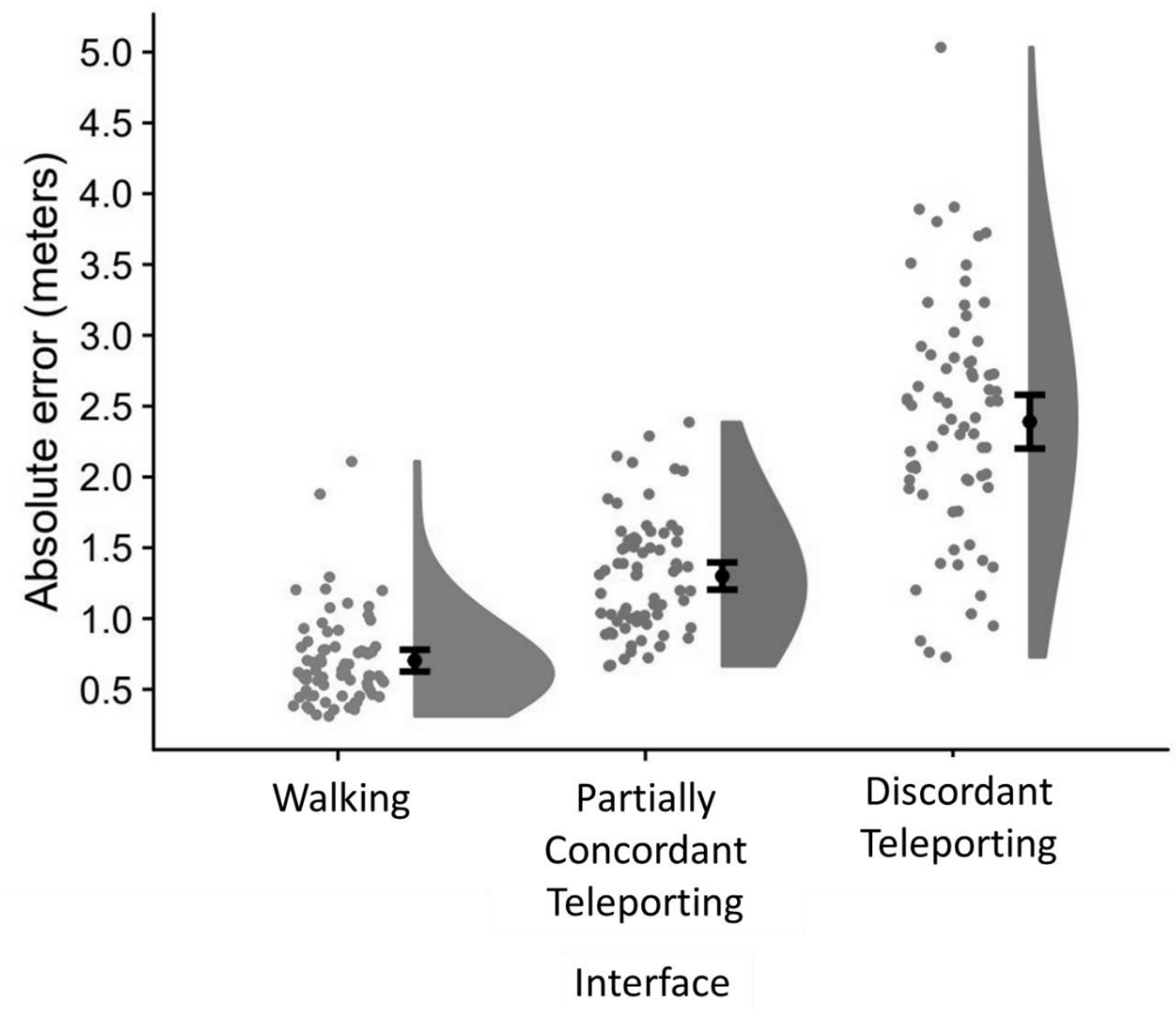

Figure 1. Raincloud plots (Allen et al., 2019) of the pooled triangle completion data from the open field VE from Cherep, Lim, Kelly, Acharya, et al. (2020) (Experiments 1-3). This graph provides individual data points, with mean and $95 \%$ confidence intervals, and density plots.

The negative influence of teleportation on triangle completion performance is robust.

Figure 1 shows means and 95\% confidence intervals (black dots with black error bars) from 72 participants pooled across multiple experiments described by Cherep, Lim, Kelly, Acharya, et al. (2020). Those data were collected using a triangle completion task in a VE that included only a grassy field without visual orienting cues, such as landmarks. When using the walking interface, participants walked and turned to travel the outbound path, and therefore received all visual and body-based cues normally associated with translation and rotation. When using the partially concordant teleporting interface, participants teleported to translate and used their bodies to 
rotate. Participants using this interface experienced no translational self-motion cues but full rotational self-motion cues. This is referred to as partially concordant because rotation through the VE was concordant with physical movement of the participant's body, whereas translation through the VE was discordant with movement of the participant's body. When using the discordant teleporting interface, participants teleported to translate and rotate, with no accompanying self-motion cues. A more complete characterization of the concordance framework for virtual locomotion can be found in Cherep, Lim, Kelly, Acharya, et al. (2020).

Although the mean differences between interfaces are large, individual data points highlight the large variability in task performance, especially for the two teleporting interfaces. This variability in individual performance suggests that not everyone is similarly impacted by the removal of self-motion cues when teleporting. The current study sought to better understand this variability by examining the relationship between triangle completion performance and characteristics of the individual.

Despite the large individual differences in the triangle completion task reported by Cherep, Lim, Kelly, Acharya, et al. (2020), there is very little research characterizing individual differences in spatial updating performance. In one of the few examples (Hegarty et al., 2002), participants were led blindfolded along an outbound path before pointing to the path origin. Pointing performance was associated with a self-reported sense of direction, such that those reporting a better sense of direction produced lower pointing errors.

Self-reported sense of direction is also associated with the ability to reorient to the environment using visual landmarks (Hegarty et al., 2002; Sholl et al., 2006), in a process known as piloting. In addition to their utility when reorienting after complete disorientation, piloting cues (e.g., landmarks) can be integrated with self-motion cues to produce more reliable estimates 
of self-location (Chen et al., 2017; Nardini et al., 2008; Sjolund et al., 2018; Zhang \& Mou, 2017; Zhang et al., 2019; Zhao \& Warren, 2015).

\section{Current Study}

The purpose of the current study was to investigate individual differences in spatial updating performance when locomotion is accomplished by walking or by teleporting. Participants performed triangle completion using three interfaces: walking, partially concordant teleporting (teleport to change position, rotate the body to change orientation), and discordant teleporting (teleport to change position and orientation). When traversing the outbound path, walking provided translational and rotational self-motion cues, partially concordant teleporting provided rotational self-motion cues only, and discordant teleporting provided no self-motion cues $^{1}$.

Furthermore, the environmental cues available in the surrounding VE were manipulated to evaluate individual differences in the integration of self-motion cues and environmental cues. The task was presented in an enclosed classroom VE replete with landmark objects (e.g., tables and chairs) which served as piloting cues, and in an open field VE with only a ground plane and sky.

In addition to the triangle completion task, participants completed several measures that might be associated with task performance. Self-report measures of sense of direction were included because they have been shown to associate with spatial updating performance (Hegarty et al., 2002). The selection of additional measures was based on details of the triangle completion

\footnotetext{
${ }^{1}$ Although discordant teleporting produced no self-motion cues, it was still possible to perform triangle completion using this interface. Specifically, the participant selected their position and orientation using the controller, and this information could be used to encode the outbound path.
} 
task and the interfaces themselves. Both forms of teleportation involve manipulation of an object in space (e.g., discordant teleporting involves positioning and orienting a marker on the ground to select the intended location and orientation). Therefore, a test of mental rotation (Vandenberg $\&$ Kuse, 1978) was included to capture this aspect of the task. The triangle completion task also involves changing perspectives when traveling along the outbound path. Therefore, a measure of perspective tasking (Kozhevnikov \& Hegarty, 2001) was included. Video game experience has also been associated with improvements in spatial cognition and perception (for a review of the relationships between video game and spatial cognition see Spence \& Feng, 2010), with some studies demonstrating relationships between experience in playing action videogames and improvements in mental rotation ability (Feng et al., 2007; Quaiser-Pohl et al., 2006). Therefore, video game experience, experience using VR, and demographic measures were also included.

Hypotheses were pre-registered prior to data collection on the Open Science Framework (doi:10.17605/OSF.IO/XC8V5). Predictions for triangle completion performance followed from previous work on the role of self-motion cues in spatial updating (Cherep, Lim, Kelly, Acharya, et al., 2020; Kelly et al., 2020; Klatzky et al., 1998; Ruddle \& Lessels, 2006). Specifically, it was expected that discordant teleporting would exhibit the highest pointing errors, followed by partially concordant teleporting, followed by walking and that this pattern would occur in both VEs. Additionally, errors were expected to be lower in the classroom VE when using the two teleporting interfaces due to the availability of piloting cues, but not when walking (mirroring the findings of Cherep, Lim, Kelly, Acharya, et al., 2020). It was also predicted that the spatial ability measures and video game experience would be significantly related to triangle completion performance. 
An exploratory latent profile analysis (LPA) was also used to examine possible group membership based on triangle completion performance. Previous studies classifying navigational performance have used cluster analysis (Weisberg \& Newcombe, 2016; Weisberg et al., 2014) which is a statistical method to find clusters or groups of observations that have similar values on a range of variables. These clusters are created so that differences within clusters are minimized while differences between clusters are maximized. The number of clusters to examine is often determined by theory or by the researcher's judgment; however, the lack of rigorous statistical methods for determining the number of clusters is often cited as a weakness of cluster analysis (Pastor et al., 2007). Therefore, for the current study, LPA was used to identify profiles (i.e., groups) of observations based on a set of continuous variables (triangle completion performance).

The purpose of an LPA is to find hidden typologies within the population. Ideally, these profiles are discrete, exhaustive, and non-overlapping. Compared with clustering analyses (e.g., k-means clustering), an advantage of LPA is that it is a model-based technique, whereas traditional cluster analyses are not (Pastor et al., 2007). Specifically, LPA is a latent variable mixture model where the term latent variable refers to the latent categorical variable of group membership and the term mixture refers to the data which are sampled from a population that contains a mix of distributions, one for each cluster, with each cluster containing its own unique set of parameters. To find the means and standard deviations of these clusters, within-class or within-cluster parameters are estimated by assigning a posterior probability. These probabilities are then used to update the within-class parameters until the parameters stop changing (for a more technical description of LPA see Oberski, 2016). In sum, LPA aims to identify homogenous groups from a heterogeneous sample by estimating profile membership 
probabilities directly from the model. Due to the exploratory nature of the LPA, there were no robust predictions regarding the number or make-up of profiles; however, following best LPA practices (Spurk et al., 2020) following the identification of profile membership based on triangle completion performance, profiles were compared on spatial ability measures, video game experience, and demographics.

The contribution of the current study is to provide a framework to describe a range of individuals who vary in susceptibility to disorientation while navigating VEs using teleportation. The results from this study could also provide avenues of future work examining how to mitigate disorientation on an individual basis. Furthermore, individual differences when navigating using teleportation may have general implications for cue integration during navigation.

\section{Method}

\section{Participants}

199 undergraduate students (97 men, 102 women) from Iowa State University participated in exchange for course credit. Data from 14 participants ( 9 men, 5 women) were removed due to missing triangle completion data for one or more cells in the experimental design, or missing spatial ability measures. In all cases, missing data was caused by equipment failure or insufficient time. Data from an additional three participants ( 2 men, 1 woman) were removed as outliers (see Results). Thus, the total sample size for the analyses was 182 (86 men, 96 women).

\section{Materials}

\section{Hardware and Software}


The HTC Vive head-mounted display presented the VEs and graphics were generated on a Windows 10 computer with an Intel 6700K processor and Nvidia GeForce GTX 1070 graphics card. Unity software displayed stereoscopic images at $1080 \times 1200$ resolution per eye with $100^{\circ}$ horizontal $\times 110^{\circ}$ vertical binocular field of view. Images refreshed at a rate of $90 \mathrm{~Hz}$ and reflected the head position and orientation tracked by the Lighthouse tracking system sold with the Vive. One wireless handheld controller, sold with the Vive, was used by participants to control the teleporting interfaces and to respond to each trial.

\section{Spatial measures and Demographics}

Santa Barbara Sense of Direction Scale. The Santa Barbara Sense of Direction scale (SBSOD) (Hegarty et al., 2002) assesses a self-report of spatial cognition and has been shown to have good internal reliability (Coefficient $\alpha=.88$ ). The SBSOD is regarded as a unitary measure of "large-scale spatial ability" which measures several different environmental-scale tasks, such as learning the layout of new environments or giving verbal navigation directions (Hegarty et al., 2006), and has been used as a measure of self-reported ability of metric knowledge of distances and directions, or "survey knowledge" (Davies, et al., 2017; Ferguson et al., 2015). The SBSOD includes 15 items that assess a participant's "sense of direction." Items are scored on a Likert scale of $(1)=$ strongly agree to $(7)=$ strongly disagree. Sample items include "I am very good at giving directions" and "I am very good at reading maps."

Philadelphia Spatial Abilities Scale. The Philadelphia Spatial Abilities Scale (PSAS) (Hegarty et al., 2010) is a self-report measure that assesses four categories of spatial tasks: static relations, relations among objects, relations within deformed objects, and relations among moving objects. The current study used a 16-item version of the scale (Hegarty et al., 2010). The PSAS has been shown to have good internal reliability (Coefficient $\alpha=.87$ ) and have good 
predictive validity for scores on tests of object transformation, such as the MRT, and a high correlation with the SBSOD. Items are scored on a Likert scale of $(1)=$ strongly agree to $(7)=$ strongly disagree. Sample items include "I can easily visualize my room with a different furniture arrangement" and "I could clearly imagine what a soda can would look like after it was partially crushed."

Mental Rotation Test. The Mental Rotation Test (MRT) (Vandenberg \& Kuse, 1978) assesses the ability to rotate mental representations of two-dimensional and three-dimensional objects in space and has a test-retest reliability of .83 . This test includes 20 items where each item consists of a criterion figure, two correct alternatives, and two incorrect or "distractor items." The correct alternatives are identical to the criterion except that each alternative has been rotated in space. Responses were scored by each accurate identification of both correct alternatives, with the maximum points possible out of 20. No points were rewarded for partial identification of one correct alternative. Participants were given six minutes to complete the test.

Spatial Orientation Test. The Spatial Orientation Test (SOT) (Hegarty \& Waller, 2004; Kozhevnikov \& Hegarty, 2001) assesses the ability to perform egocentric perspective transformations and has been shown to have good internal reliability (Coefficient $\alpha=.83$ ). This test presents the participant with an overhead view of an object array. With the array in view, the participant is asked to imagine standing at one object, facing a second object, and point to a third object from the imagined perspective. Traditionally, the pointing response is executed by drawing a radial line through a circle to indicate the egocentric direction of the third object relative to the imagined perspective. In the current study, this measure was modified for online administration. Rather than drawing the directional response, the participant viewed a circle numbered in minutes (1-60) and selected the number corresponding to the egocentric direction of 
the third object. Performance on a computerized version of the SOT is similar to the original paper-based version (Friedman et al., 2019). For scoring, responses were converted from minutes to degrees, then the correct answer was subtracted from the participant's response. Absolute errors were then calculated, and if the absolute error exceeded $180^{\circ}$ it was subtracted from $360^{\circ}$. Final errors ranged from $0^{\circ}$ to $180^{\circ}$, with lower errors indicating better performance. Participants were given five minutes to complete the test.

Video Games. Participants were asked to estimate how many hours they play video games per weekday and per weekend day in the last calendar year. Each estimate was multiplied by five and two, respectively, and then summed to yield weekly video game hours. Participants also reported the genre of video games, including VR games. Experience with VR games was coded as $0=$ no experience or $1=$ experience with VR.

Demographics. Gender was recorded by the experimenter for each participant and was coded as $0=$ men and $1=$ women .

\section{Stimuli}

\section{Virtual environments}

The experiment included three VEs: the training VE, the open field VE, and the classroom VE. The training VE contained a grid-like ground texture and no landmarks. The open field VE consisted of an infinite ground plane with grass texture and blue sky (Figure 2, top). The classroom VE was based on a real classroom at Iowa State University (Figure 2, bottom). The walls of the 3D model were textured with photographs from the real classroom. The classroom VE was square with $9.14 \mathrm{~m}$ sides and included several 3D models of classroom furniture such as chairs, tables, and a classroom media console. 

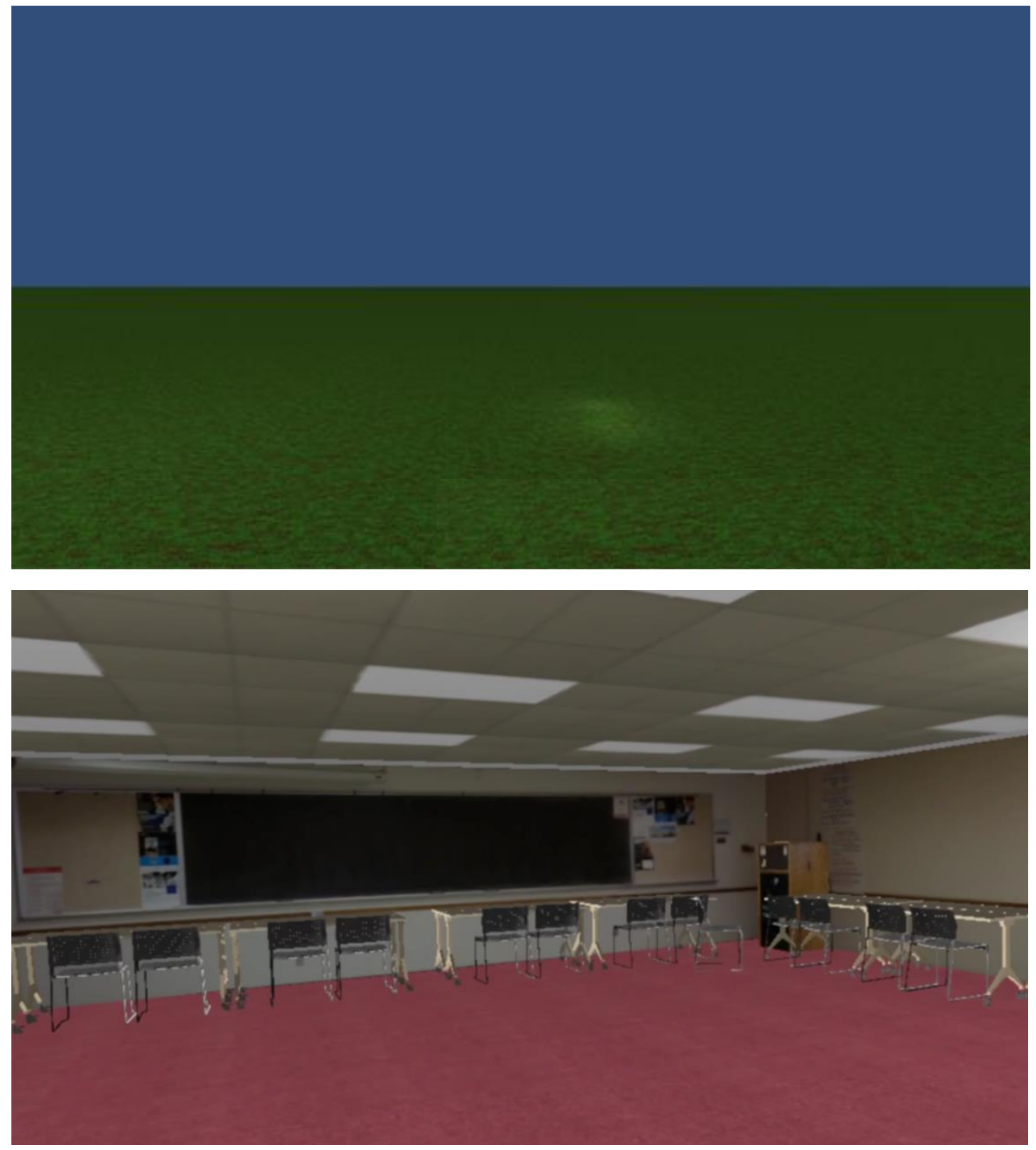

Figure 2. Image of the two experimental VEs: open field VE (top) and classroom VE (bottom).

Three color-coded posts indicated each vertex of the triangular path: a green post marked the path origin, a yellow post indicated the end of the first path leg, and a red post denoted the end of the second path leg. A white arrow located at the bottom of the first and second posts indicated the orientation of the subsequent path leg. For all locomotion interfaces, a virtual replica of the handheld controller was visible to the participant. 


\section{Interfaces}

For walking, the participant physically walked and rotated to change position and orientation.

To teleport with the partially concordant interface, the participant selected a location on the ground plane by pressing down on the controller's touchpad, which produced a white circle (30 cm diameter) surrounded by a white ring $(75 \mathrm{~cm}$ diameter) on the ground plane (see Figure 3 , top). This white teleport marker was connected to the controller by a red line, and the participant controlled the position of the maker as if aiming a laser pointer. Upon the release of the touchpad, the participant was instantly teleported to the new position. The participant's previous orientation was preserved until the participant physically rotated to face the next post in the outbound path. 

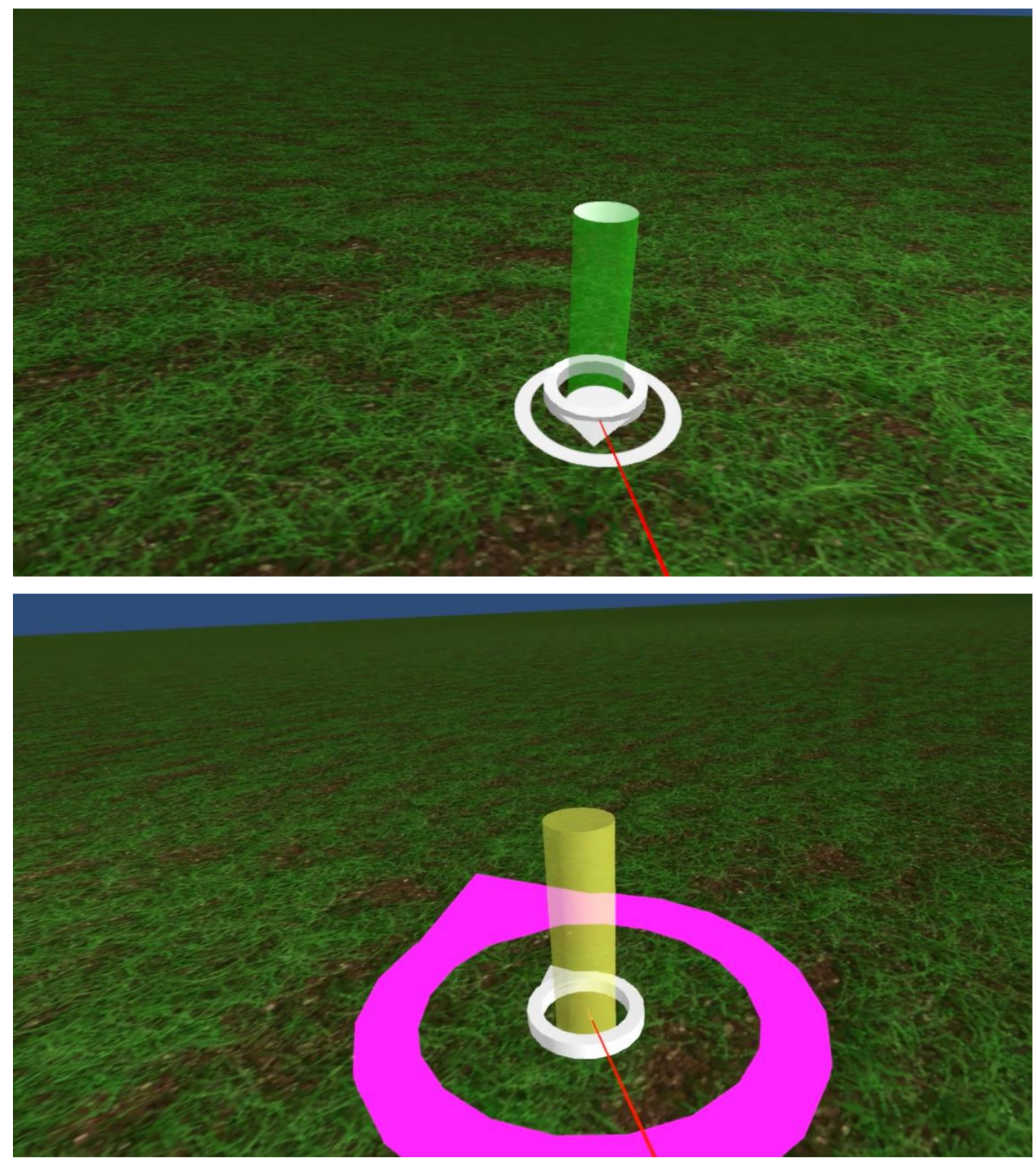

Figure 3. The two teleporting interfaces, shown in the open field VE: partially concordant teleporting (top), in which the user controlled the white ring's location, and discordant teleporting (bottom), in which the user controlled the magenta ring's location and orientation. Also visible in the images is the white arrow at the base of each post, indicating the direction of the next post.

To teleport with the discordant interface, the participant selected a location and orientation on the ground plane by positioning and orienting an arrow affixed to a magenta ring (height $=7.50 \mathrm{~cm}$ diameter; outer diameter $=195 \mathrm{~cm}$ diameter $)$, which indicated the intended 
position and orientation (see Figure 3, bottom). To change position and orientation, the participant simultaneously held down the Vive controller touchpad and positioned their thumb on the edge of the touchpad to adjust the orientation of the teleportation arrow. For example, a thumb position directly to the left of the center of the touchpad corresponds to $90^{\circ}$ counterclockwise rotation from the participant's current facing direction while a thumb position directly beneath the center of the touchpad corresponds to a $180^{\circ}$ rotation from the participant's current facing direction. Upon the release of the touchpad, the participant was instantly teleported to the new position and orientation.

Upon reaching the end of the path, the participant pointed to the path origin by pressing down on the Vive controller trigger to display a blue disk $(39 \mathrm{~cm}$ diameter) on the ground plane. The blue disk was connected to the controller by a red line, and the participant controlled the position of the disk as if aiming a laser pointer. The response was instantly recorded upon the release of the trigger.

\section{Design}

The triangle completion experiment employed a 2 (VE: open field vs. classroom) x 3 (Interface: walking vs. partially concordant teleporting vs. discordant teleporting) repeatedmeasures design. Participants also completed performance-based and self-report spatial ability measures and surveys of weekly average of video gameplay and previous VR experience. Demographic information was also recorded.

For each combination of VE and interface, participants completed a block of 12 triangle completion trials corresponding to 12 turn angles (left and right turns of 22.50, 45, 67.50, 90, 112.50 , and $135^{\circ}$ ). Trial order was randomized within blocks. Path leg length was randomly 
selected on each trial from three possible options (first path leg: 1.52, 1.68, or $1.83 \mathrm{~m}$; second path leg: $1.22,1.37$, or $1.52 \mathrm{~m}$ ). The path origin was selected from eight possible locations evenly distributed around the VE to ensure that participants would not be required to navigate outside the tracked space when walking. Path origin positions were pseudo-randomized with the constraint that the same path origin was not repeated twice in a row (to ensure that participants did not receive feedback about their responses).

The VE variable was blocked, such that participants completed all three interfaces in one VE and then completed all three interfaces in the other VE, and VE order was counterbalanced. Interface order was counterbalanced and repeated across the two VE blocks.

The primary dependent variable in the triangle completion task was absolute pointing error, calculated as the absolute distance (in meters) between the path origin and the participant's response. Response latency, the time between arrival at the red post and response, was also recorded. Only absolute distance errors are presented, but supplemental analyses with response latency can be found on the Open Science Framework (doi:10.17605/OSF.IO/XC8V5).

\section{Procedure}

After signing the informed consent, the participant was given verbal instructions on the triangle completion task. The participant then donned the head-mounted display and was trained on the triangle completion task in the training VE with each of the locomotion interfaces. The participant was required to complete three practice trials with each locomotion interface and could request additional practice. Experimental trials began after training completion. Performance-based feedback was not provided. 
On each triangle completion trial, the participant traveled the outbound path by moving to the green post (the path origin), then the yellow post, then the red post. Posts were presented sequentially and disappeared upon arrival. Once all six triangle completion blocks were complete, the participant sat in front of a lab computer and completed the spatial ability measures and demographic information. After completing the experiment, the participant was debriefed and given course credit. The study typically lasted around 90 minutes.

\section{Results}

Possible multivariate outliers were examined and analyzed for the six triangle completion conditions (open field: walking, partially concordant teleporting, and discordant teleporting; classroom: walking, partially concordant teleporting, discordant teleporting) using Mahalanobis distance. Possible outliers were compared against the allotted distance from the determined group center statistic $\left(\chi^{2}=22.46\right.$ for $\left.\mathrm{df}=6, p<.001\right)$. There were two extreme cases ( 89.10 and 60.91), and these participants were removed from the data set (1 man and 1 woman). Finally, one participant (1 man) was removed for reporting weekly video game hours greater than three standard deviations from the group mean.

\section{Spatial Measure Reliabilities and Correlations}

Internal consistency was good for the MRT (Kuder-Richardson $20=.81$ ), the SBSOD (Coefficient $\alpha=.87$ ), the SOT (Coefficient $\alpha=.87$ ), and the PSAS (Coefficient $\alpha=.81$ ). Bivariate correlations, means, and standard deviations are presented in Table 1. Relationships between the spatial measures were similar to those reported in previous studies (e.g., Hegarty \& Waller, 2004; Hegarty et al., 2010). The negative associations with SOT are expected because performance on that measure was coded as degrees of error. The MRT was significantly 
associated with the $\operatorname{SBSOD}(r=.32, p<.01)$, the SOT $(r=-.65, p<.01)$, the PSAS $(r=.26, p<$ $.01)$, and weekly video gameplay $(r=.26, p<.01)$. The SBSOD was significantly associated with the SOT $(r=-.34, p<.01)$, and the PSAS $(r=.40, p<.01)$, but was not significantly related to weekly video gameplay (in hours, $r=.10$ ). The SOT was significantly associated with the PSAS $(r=-.30, p<.01)$ and hours of video gameplay per week $(r=-.27 . p<.01)$. Gender was positively associated with triangle completion errors, such that women (coded as 1 and men as 0) tended to produce higher errors during partially concordant teleporting (open field: $\mathrm{r}=.26, p<$ .01 ; classroom: $\mathrm{r}=.39, p<.01$ ) and discordant teleporting (open field: $\mathrm{r}=.36, p<.01$; classroom: $\mathrm{r}=.30, p<.01)$ in both VEs compared to men. Gender was also associated with the spatial ability measures as women tended to perform worse on the MRT $(r=-.44, p<.01)$, the $\operatorname{SBSOD}(r=-.25, p<.01)$, the SOT $(r=.43, p<.01)$, and the PSAS $(r=-.19, p<.01)$. Women also reported fewer video game hours per week $(r=-.35, p<.01)$.

Triangle completion error across the six conditions was also significantly intercorrelated. Although triangle completion error in the walking open field and classroom conditions had no significant correlations with any spatial ability measures, the teleporting conditions did have significant associations with spatial ability measures and participant characteristics. These relationships are explored more fully in the LPA. 
Table 1. Means, standard deviations, and correlations between study variables

\begin{tabular}{|c|c|c|c|c|c|c|c|c|c|c|c|c|c|}
\hline & 1. & 2. & 3. & 4. & 5. & 6. & 7. & 8. & 9. & 10. & 11. & $M$ & $S D$ \\
\hline 1. Gender & --- & & & & & & & & & & & --- & --- \\
\hline 2. Field - Walking & -.05 & --- & & & & & & & & & & .71 & .25 \\
\hline $\begin{array}{l}\text { 3. Field - Partially } \\
\text { Concordant }\end{array}$ & $.26 * *$ & $.32 * *$ & --- & & & & & & & & & 1.38 & .46 \\
\hline 4. Field - Discordant & $.36 * *$ & $.19 * *$ & $.55^{* *}$ & --- & & & & & & & & 2.33 & .78 \\
\hline $\begin{array}{l}\text { 5. Classroom - } \\
\text { Walking }\end{array}$ & -.02 & $.36 * *$ & $.25 * *$ & .13 & --- & & & & & & & .64 & .20 \\
\hline $\begin{array}{l}\text { 6. Classroom - } \\
\text { Partially Concordant }\end{array}$ & $.39 * *$ & $.16^{*}$ & $.38 * *$ & $.39 * *$ & $.23 * *$ & --- & & & & & & .94 & .37 \\
\hline $\begin{array}{l}\text { 7. Classroom - } \\
\text { Discordant }\end{array}$ & $.30 * *$ & $.22 * *$ & $.37 * *$ & $.38 * *$ & $.20 * *$ & $.74 * *$ & --- & & & & & 1.33 & .70 \\
\hline 8. MRT & $-.44 * *$ & -.12 & $-.35 * *$ & $-.36 * *$ & -.10 & $-.44 * *$ & $-.44 * *$ & --- & & & & 9.69 & 4.69 \\
\hline 9. SBSOD & $-.25 * *$ & -.07 & -.09 & $-.17 *$ & -.08 & $-.20 * *$ & $-.17 *$ & $.32 * *$ & --- & & & 4.15 & 1.02 \\
\hline 10. SOT & $.43 * *$ & .13 & $.32 * *$ & $.32 * *$ & .02 & $.56^{* *}$ & $.46^{* *}$ & $-.65^{* *}$ & $-.34 * *$ & --- & & 36.90 & 27.00 \\
\hline 11. PSAS & $-.19 * *$ & -.10 & $-.17 *$ & -.07 & .05 & $-.23 *$ & $-.17 *$ & $.26^{*}$ & $.40^{* *}$ & $-.30 * *$ & --- & 4.54 & .80 \\
\hline $\begin{array}{l}\text { 12. Weekly video } \\
\text { gameplay (hours) }\end{array}$ & $-.35 * *$ & .08 & -.09 & $-.15^{*}$ & .11 & $-.25 * *$ & $-.22 * *$ & $.26 * *$ & .10 & $-.27 * *$ & -.10 & 13.58 & 13.17 \\
\hline
\end{tabular}

Note. Gender was coded $0=$ men and $1=$ women.

$* p<.05 . * * p<.01$ 


\section{Effects of Environment and Interface on Triangle Completion}

There was no evidence of a speed-accuracy trade-off on the triangle completion task as the within-participant correlation between absolute pointing error and latency was significantly positive $(M=.31, S E=.04), t(181)=8.34, p<.001$. Absolute errors (Figure 4$)$ were analyzed in a repeated-measures ANOVA with terms for interface and environment. Mauchly's Test of Sphericity indicated that the assumption of sphericity had been violated for interface, $\chi^{2}(2)=$ 103.85, $p<.001$, and the interaction term, $\chi^{2}(2)=63.74, p<.001$. Therefore, a Huynh-Feldt correction and a Greenhouse-Geisser correction was used for interface and the interaction term, respectively. Significant main effects for interface, $F(1.40,253)=563.80, p<.001, \eta^{2}{ }_{p}=.63$, and environment, $F(1,181)=304.04, p<.001, \eta_{p}^{2}=.76$, were qualified by a significant interaction between interface and environment, $F(1.54,278.85)=150.22, p<.001, \eta^{2} p=.45$. 


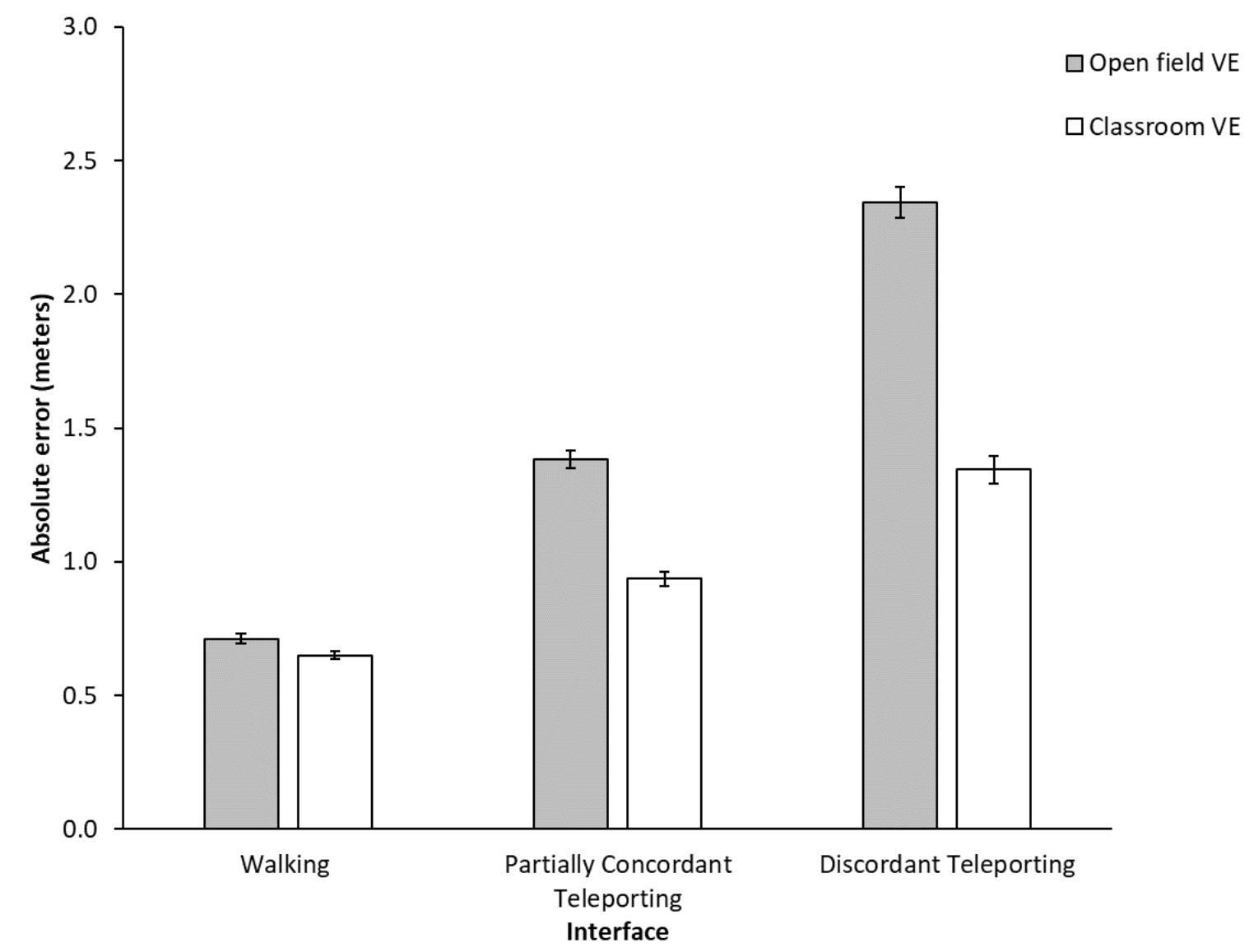

Figure 4. Average absolute error when performing the triangle completion task. Error bars represent $\pm 1 S E M$.

Across both environments, the discordant teleporting interface produced larger errors than the partially concordant interface; classroom: $t(181)=11.10, p<.001, d=.81$; open field: $t(181)=19.55, p<.001, d=1.45$ (Figure 4$)$. The partially concordant interface produced larger errors than the walking interface; classroom: $t(181)=9.98, p<.001, d=.76$; open field: $t(181)=$ 20.22, $p<.001, d=1.50$. Additionally, the classroom VE produced lower errors compared to the open field when using the discordant teleporting interface, $t(181)=16.10, p<.001, d=1.20$, the partially concordant teleporting interface, $t(181)=12.86, p<.001, d=.94$, and the walking interface, $t(181)=3.12, p=.002, d=.23$.

\section{Latent Profile Analysis}


As stated previously, the purpose of an LPA is to identify group membership composed of different levels from a set of variables. A major advantage of using LPA over traditional cluster techniques is the numerous statistical criteria (fit indices) provided which aid in deciding upon the final number of profiles. The most common fit indices include the log-likelihood (LL), Akaike information criteria (AIC), the Bayesian information criteria (BIC), the sample-size adjusted BIC (SSA BIC), the Lo-Mendell-Rubin adjusted likelihood ratio (LMRT), the bootstrap likelihood ratio (BLRT), and associated $p$-values. When examining fit indices, lower values indicate better model fit, although only certain fit indices, such as LMRT and BLRT, provide a $p$-value to test for a significantly better model fit (Oberski, 2016). The way to interpret model fit is to compare $K-1$, where $K$ is the number of profiles. Although fit indices provide a rigorous statistical way for researchers to examine model fit, as with exploratory factor analyses, it is also recommended that the profiles be examined with consideration of theory, sample size, ease of interpretation, and the uniqueness and meaningfulness of the profiles (Gabriel et al., 2015; Hirschi \& Valero, 2017; Spurk et al., 2020; Vermunt \& Magidson, 2002; Woo et al., 2018). For the current study, only the AIC, BIC, entropy, and profile sizes are presented in Table 2. Additional fit indices can be found on the Open Science Framework (doi:10.17605/OSF.IO/XC8V5).

Triangle completion data from six conditions (three interfaces $\times$ two VEs) were used to identify latent profiles using Mplus version 7 (Muthén \& Muthén, 1998-2012). As fit indices within the data suggested different profile counts, interpretability and utility were considered when deciding on the final profile count (see Table 2). Although the four-profile model produced better model fit (lower AIC and BIC values) the size of each profile should also be taken into account. When profile sizes are small, it begs the question of whether the additional profile 
outweighs the concerns of lower power, lower precision to detect meaningful differences amongst other variables, and less parsimony. Suggested size thresholds for rejected profiles have included whether the profile has $<1.0 \%$ of the total sample size or contains fewer than 25 individuals (Lubke \& Neale, 2006). Therefore, the additional profile from the four-profile solution was determined too small to be meaningful $(n=8)$ and ease of interpretability and parsimony suggested a three-profile solution. Further support for the three-profile model comes from the entropy value (.84). Entropy values around .76 and above are related to an accuracy of 90\% in correctly assigning profile membership to participants (Wang et al., 2017) which suggests that individuals were classified with a high degree of accuracy in the three-profile model.

Table 2. Fit statistics for the latent profile analysis of triangle completion performance

\begin{tabular}{|c|c|c|c|c|c|c|c|}
\hline Model & \multicolumn{7}{|c|}{ Fit Indices } \\
\hline $\begin{array}{l}\text { Number of } \\
\text { Profiles }\end{array}$ & AIC & BIC & Entropy & 1 & 2 & 3 & 4 \\
\hline 1 & 1196.160 & 1234.608 & 1.000 & $\begin{array}{c}182 \\
(100 \%)\end{array}$ & & & \\
\hline 2 & 997.361 & 1058.237 & 0.793 & $\begin{array}{c}95 \\
(52 \%)\end{array}$ & $\begin{array}{c}87 \\
(48 \%)\end{array}$ & & \\
\hline 3 & 892.769 & 976.073 & 0.835 & $\begin{array}{c}76 \\
(42 \%)\end{array}$ & $\begin{array}{c}72 \\
(40 \%)\end{array}$ & $\begin{array}{c}34 \\
(18 \%)\end{array}$ & \\
\hline 4 & 838.215 & 946.947 & 0.885 & $\begin{array}{c}82 \\
(45 \%)\end{array}$ & $\begin{array}{c}66 \\
(35 \%)\end{array}$ & $\begin{array}{c}26 \\
(15 \%)\end{array}$ & $\begin{array}{c}8 \\
(5 \%)\end{array}$ \\
\hline
\end{tabular}

Note . AIC $=$ Akaike Information Criteria; BIC $=$ Bayesian Information Criteria. The full fit indices can be found on the Open Science Framework (Supplemental Figure S14).

Triangle completion error is shown separately for each profile in Figure 5 (for a bar graph version with error bars, see Supplemental Figure S1). All three profiles contained a reasonable proportion of the sample and varied in a distinct, identifiable pattern across the different variables manipulated in our study. After inspecting each profile, labels should be assigned to 
single profiles (Spurk et al., 2020). The labels selected are up to the discretion of the researcher but should be related to the variables and respective levels in the LPA or capture the general theme of the profiles. One profile, termed "Accurate Integrators", had low triangle completion errors across all conditions and performed better in the classroom VE than the open field VE $(42 \%, n=76)$, which suggests that they integrated self-motion cues with landmarks when available. Another profile, termed "Inaccurate Integrators", also performed better in the classroom VE than the open field VE but had higher overall errors $(40 \%, n=72)$. The final profile, termed "Inaccurate Non-Integrators", had high errors and improved little when landmarks were available $(18 \%, n=34)$. 


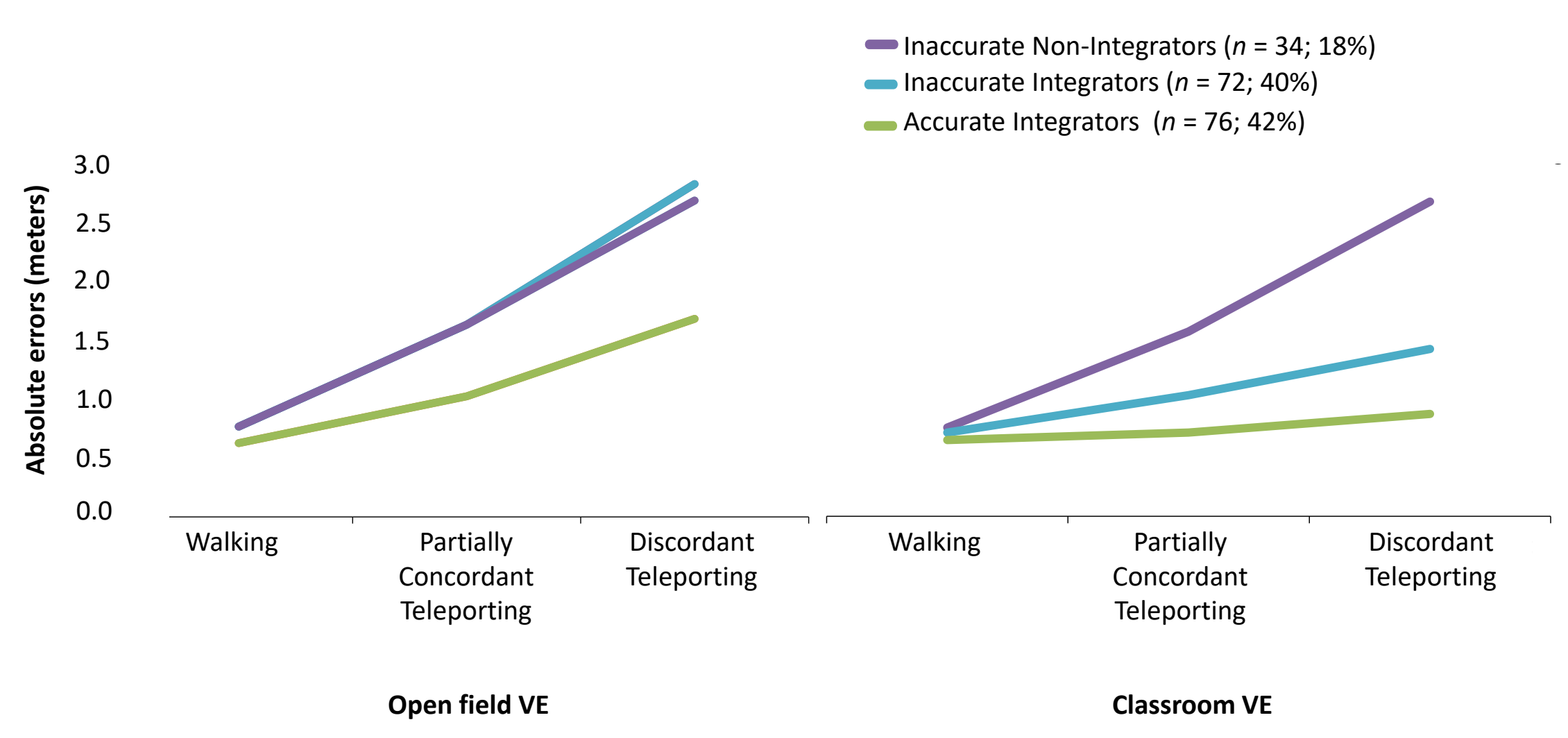

Figure 5. Three-profile model of triangle completion performance. 


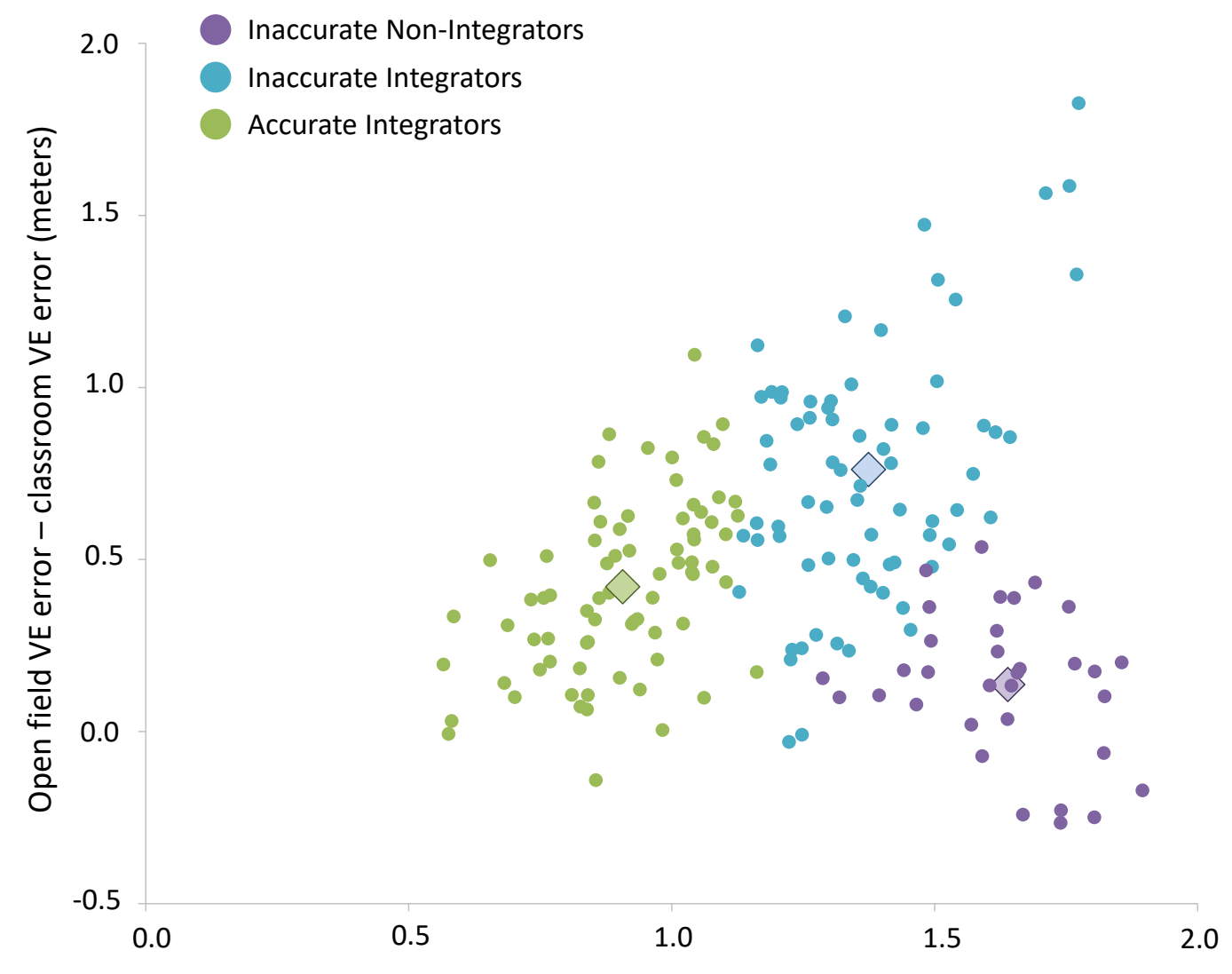

Average absolute error (meters) across conditions

Figure 6. Scatterplot of individual performance grouped by profile membership on the triangle completion task. Colored diamonds are the profile average.

Figure 6 shows how the profile labels reflect triangle completion errors, specifically overall accuracy (shown on the $\mathrm{x}$-axis as mean error across the six conditions) and integration of self-motion and environmental cues (shown on the y-axis as the mean difference in performance between the open field VE and the classroom VE; larger values reflect the greater benefit of the classroom). Note that the data shown in Figure 6 are presented to justify the profile labels and that the data in Figure 5 were used to compute the profiles themselves. Accurate Integrators cluster toward the left of the figure due to their overall good performance, and are generally 
above zero on the y-axis indicating that they integrate self-motion cues with environmental cues available in the classroom VE. Inaccurate Integrators are shifted to the right relative to accurate integrators due to their larger overall errors, and they are also generally above zero on the y-axis indicating integration of self-motion and environmental cues. Integration is more apparent (higher on the y-axis) among Inaccurate Integrators compared to Accurate Integrators, probably because Inaccurate Integrators performed worse in the open field and therefore had more room to improve in the classroom. Inaccurate Non-Integrators are shifted to the right relative to the two other groups indicating large overall errors, and they are evenly distributed around zero on the yaxis indicating a lack of integration of environmental cues.

\section{Triangle Completion Performance Differences}

Following the LPA, the three profiles were examined using one-way ANOVAs and posthoc comparisons to identify any significant differences in triangle completion errors across the six VR conditions. Levene's test showed that the variances for all of the classroom conditions and the partially concordant and discordant open field conditions were not equal ( $p$ 's $<.01)$. Therefore, the Brown-Forsythe statistic was reported for all of the classroom conditions and the partially concordant field conditions since the data were positively skewed. The Welch statistic was used for the discordant field conditions since the data were not skewed. For post-hoc comparisons, the Games-Howell correction was applied to all conditions to account for unequal variances and sample size, except for the walking interface in the open field VE which used Hochberg's GT2.

In the open field VE, Accurate Integrators performed better than Inaccurate Integrators and Inaccurate Non-Integrators on all three interfaces, and Inaccurate Integrators and Inaccurate Non-Integrators did not significantly differ on any interface. The main effect of profile was 
significant when using the walking interface, $F(2,179)=7.34, p=.001, \eta^{2}{ }^{2}=.08$, such that Accurate Integrators $(M=.63, S D=.22)$ had significantly lower errors compared to Inaccurate Integrators $(M=.77, S D=.23, p=.002)$ and Inaccurate Non-Integrators $(M=.77, S D=.31, p=$ .015). Inaccurate Integrators were not significantly different from Inaccurate Non-Integrators ( $p$ $=1.00$ ). The main effect of profile was also significant when using the partially concordant teleporting interface, $F(2,149.41)=77.12, p<.001, \eta^{2} p=.51$. Accurate Integrators $(M=1.03$, $S D=.27)$ had lower errors compared to Inaccurate Integrators $(M=1.64, S D=.45, p<.001)$ and Inaccurate Non-Integrators $(M=1.64, S D=.24, p<.001)$. There was not a significant difference between Inaccurate Integrators and Inaccurate Non-Integrators $(p=.994)$. Finally, the main effect of profile was also significant when using the discordant teleporting interface, $F(2,179)=$ 7.34, $p<.001, \eta^{2}{ }^{2}=.08$. Accurate Integrators $(M=1.69, S D=.53)$ had significantly lower errors compared to Inaccurate Integrators $(M=2.84, S D=.67, p<.001)$ and Inaccurate NonIntegrators $(M=2.70, S D=.37, p<.001)$. There was not a significant difference between Inaccurate Integrators and Inaccurate Non-Integrators $(p=.292)$.

Analysis of the errors in the classroom VE showed that the three profiles were indistinguishable when walking, but that partially concordant and discordant teleporting led to lower errors for Accurate Integrators compared to Inaccurate Integrators, and lower errors for Inaccurate Integrators compared to Inaccurate Non-Integrators. The main effect of profile was significant when using partially concordant teleporting, $F(2,68.95)=109.15, p<.001, \eta^{2} p=.76$. Accurate Integrators $(M=.67, S D=.17)$ had significantly better performance using partially concordant teleporting compared to Inaccurate Integrators $(M=.97, S D=.22, p<.001)$ and Inaccurate Non-Integrators $(M=1.48, S D=.34, p<.001)$, and Inaccurate Integrators had significantly better performance compared to Inaccurate Non-Integrators $(p<.001)$. There was 
also a main effect of profile for discordant teleporting in the classroom VE, $F(2,84.24)=232.22$, $p<.001, \eta^{2}{ }_{p}=.85$. Accurate Integrators $(M=.82, S D=.24)$ had significantly better performance compared to Inaccurate Integrators $(M=1.34, S D=.38, p<.001)$ and Inaccurate NonIntegrators $(M=2.52, S D=.46, p<.001)$, and Inaccurate Integrators had significantly better performance compared to Inaccurate Non-Integrators $(p<.001)$. There was no significant main effect of profile for walking in the classroom VE, $F(2,70.35)=2.63, p=.079, \eta^{2} p=.07$. Accurate Integrators $(M=.61, S D=.18)$ were not significantly different from Inaccurate Integrators $(M=.67, S D=.18, p=.073)$, or Inaccurate Non-Integrators $(M=.71, S D=.31, p=$ .167). There was no significant difference between Inaccurate Integrators and Inaccurate NonIntegrators for walking in the classroom VE either $(p=.777)$.

Collapsing across interface, there were significant differences for the three profiles across VEs. Accurate Integrators showed significant improvement in triangle completion errors in the classroom $(M=.74, S D=.17)$ compared to the open field $(M=1.36, S D=.33), t(75)=15.10, p$ $<.001, d=1.74$. Inaccurate Integrators also showed significant improvement in the classroom $(M=1.16, S D=.25)$ compared to the open field $(M=2.25, S D=.43), t(71)=16.46, p<.001, d$ $=2.60$, as did the Inaccurate Non-Integrators; classroom $(M=2.00, S D=.28)$, open field $(M=$ $2.18, S D=.22) t(33)=3.22, p=.003, d=0.54$. Notably, although the Inaccurate Non-Integrators showed improvement in the classroom VE this difference was much smaller $(d=.54)$ compared to the other two profiles; Accurate Integrators $(d=1.74)$ and Inaccurate Integrators $(d=2.60)$.

To summarize, Accurate Integrators had significantly lower absolute distance errors compared to the Inaccurate and Inaccurate Non-Integrators across all conditions except the classroom walking condition. Inaccurate Integrators and Inaccurate Non-Integrators performed similarly in the open field VE, but Inaccurate Integrators performed better than Inaccurate Non- 
Integrators in the classroom VE when using the partially concordant and discordant teleporting interfaces.

\section{Demographic Differences}

The three profiles were also examined to determine whether there were significant differences in gender, weekly video game hours, and VR experience (Table 3). For gender, significant differences emerged, $\chi^{2}(2)=27.63, p<.001$, such that there were more women $(65.3 \%, n=47)$ than men $(34.7 \%, n=25)$ in the Inaccurate Integrators profile and more women $(76.5 \%, n=26)$ than $(23.5 \%, n=8)$ men in the Inaccurate Non-Integrators profile. For the Accurate Integrator profile, there were more men $(69.7 \%, n=56)$ than women $(30.3 \%, n=23)$. Profiles also significantly differed on weekly video game hours (Supplemental Figure S2), $F(2$, $146.04)=5.58, p=.005, \eta^{2}=.07$. Accurate Integrators reported significantly more video game hours $(M=17.24, S D=13.77)$ than Inaccurate Integrators $(M=11.31, S D=12.26, p=.017)$ and Inaccurate Non-Integrators $(M=10.21, S D=11.99, p=.026)$. Inaccurate Integrators and Inaccurate Non-Integrators did not significantly differ on video game hours $(p=.968)$. Profiles also significantly differed in VR experience, $\chi^{2}(2)=8.39, p=.015$, as eleven participants in the Accurate Integrators profile (73.33\%) reported having used VR previously, compared to one participant in the Inaccurate Integrators profile (6.67\%) and three participants in the Inaccurate Non-Integrators profile $(20 \%)$. 
Table 3. Demographic differences in spatial abilities for the three-profile model

\section{Latent Profiles}

\begin{tabular}{lccc}
\hline & $\begin{array}{c}\text { Accurate Integrators } \\
n=76(42 \%)\end{array}$ & $\begin{array}{c}\text { Inaccurate Integrators } \\
n=72(40 \%)\end{array}$ & $\begin{array}{c}\text { Inaccurate Non-Integrators } \\
n=34(18 \%)\end{array}$ \\
\hline $\begin{array}{l}\text { Weekly video } \\
\text { gameplay (hours) }\end{array}$ & $17.24(13.77)_{\mathrm{a}}$ & $11.31(12.26)_{\mathrm{b}}$ & $10.21(11.99)_{\mathrm{b}}$ \\
Gender & $\begin{array}{c}\text { Men }=56(69.7 \%) ; \\
\text { Women }=23(30.3 \%)\end{array}$ & $\begin{array}{c}\text { Men }=25(34.7 \%) ; \\
\text { Women }=47(65.3 \%)\end{array}$ & $\begin{array}{c}\text { Men }=8(23.5 \%) ; \\
\text { Women }=26(76.5 \%)\end{array}$ \\
VR experience & $11(73.33 \%)$ & $1(6.67 \%)$ & $3(20 \%)$ \\
\hline
\end{tabular}

Note. For weekly video gameplay, means and standard deviations (in parentheses) are shown. Means that do not share the same subscripts differ by $p<.05$ based on post hoc pairwise comparisons. For gender and VR experience, the number of individuals and percentage (in parentheses) are shown. Statistics can be found under the demographic differences heading.

\section{Spatial Measure Differences}

One-way ANOVAs and post-hoc comparisons were conducted to examine whether performance-based measures of spatial ability (MRT and SOT) and self-report measures of spatial ability (SBSOD and PSAS) significantly differed among the three profiles. Levene's test showed that the variances for the SBSOD, $F(2,179)=3.10, p=.048$, and the SOT, $F(2,179)=$ 26.13, $p<.001$ were not equal. Therefore, the Brown-Forsythe statistic was reported for the SOT since the data were positively skewed. Since the data were not skewed for the SBSOD, the Welch statistic was used. The Games-Howell post-hoc correction was applied to the SBSOD and the SOT to account for unequal variances and sample size, and Hochberg's GT2 post-hoc correction was used for the MRT and the PSAS.

For the performance-based spatial ability measures (see Supplemental Figures S3 and S4), there was a significant main effect of profile, $F(2,91.51)=27.23, p<.001, \eta^{2}{ }^{2}=.37$. 
Accurate Integrators $(M=22.45, S D=13.38)$ performed significantly better (i.e., lower error) on the SOT compared to Inaccurate Integrators $(M=41.57, S D=28.15, p<.001)$ and Inaccurate Non-Integrators $(M=59.31, S D=29.00, p<.001)$. There was also a significant difference between Inaccurate Integrators and Inaccurate Non-Integrators $(p=.012)$. For the MRT there was also a significant main effect of profile, $F(2,179)=24.71, p<.001, \eta^{2}{ }^{2}=.22$, where Accurate Integrators $(M=12.11, S D=4.18)$ performed significantly better on the MRT compared to Inaccurate Integrators $(M=8.64, S D=4.13, p<.001)$ and Inaccurate NonIntegrators $(M=6.53, S D=4.29, p<.001) .$. There was a marginally significant difference between Inaccurate Integrators and Inaccurate Non-Integrators $(p=.048)$.

For the subjective (i.e., self-report) spatial ability measures (see Supplemental Figures S5 and S6), there was a significant main effect of profile on the SBSOD, $F(2,84.68)=4.84, p=$ $.010, \eta^{2}{ }_{p}=.10$. Accurate Integrators $(M=4.40, S D=1.11)$ reported significantly better perceived sense of direction on the SBSOD compared to Inaccurate Non-Integrators $(M=3.72, S D=1.08$, $p=.010)$, but not with Inaccurate Integrators $(M=4.08, S D=.80, p=.110)$. There were no significant differences between Inaccurate Integrators and Inaccurate Non-Integrators on the $\operatorname{SBSOD}(p=.202)$. For the PSAS, there was also a significant main effect of profile, $F(2,179)=$ $3.52, p=.032, \eta^{2} p=.04$. Accurate Integrators $(M=4.69, S D=.84)$ reported significantly greater perceived small-scale spatial ability on the PSAS compared to Inaccurate Non-Integrators $(M=$ $4.54, S D=.80, p=.028)$, but not with Inaccurate Integrators $(M=4.51, S D=.80, p=.444)$. There were no significant differences between Inaccurate Integrators and Inaccurate NonIntegrators on the PSAS $(p=.332)$.

\section{Gender Differences}


The three profiles differed significantly in gender proportion. Specifically, there were more men than women in the Accurate Integrators profile, but more women than men in the Inaccurate Integrators and Inaccurate Non-Integrators profiles. Gender has long been a variable of interest in spatial cognitive research; therefore, independent sample t-tests were conducted to investigate differences between men and women for triangle completion errors across the six conditions (see Supplemental Figure S7). Men, compared to women, had significantly lower absolute distance error while using partially concordant teleportation in the open field VE, $t(180)$ $=3.53, p=.001, d=.52$, men $(M=1.26, S D=.50)$, women $(M=1.49, S D=.39)$, and in the classroom VE, $t(168.40)=5.82, p<.001, d=.84$, men $(M=.78, S D=.27)$, women $(M=1.07$, $S D=.40)$. Men also had significantly lower errors while using discordant teleporting in the open field VE, $t(155.20)=5.09, p<.001, d=.77$, men $(M=2.04, S D=.85)$, women $(M=2.61, S D=$ $.63)$, and in the classroom VE, $t(179.64)=4.32, p=.001, d=.64$, men $(M=1.12, S D=.62)$, women $(M=1.55, S D=.72)$. There were no significant differences between men and women for walking in the open field VE, $t(180)=.65, p=.518, d=.08$, men $(M=.72, S D=.27)$, women $(M=.70, S D=.23)$, or for the classroom VE, $t(180)=.26, p=.792, d=.05$, men $(M=.66, S D=$ $.22)$, women $(M=.65, S D=.21)$. In summary, men tended to have lower absolute distance errors across both teleportation interfaces in each VE, but men and women performed similarly when walking.

Independent sample t-tests also showed that men compared to women had significantly better performance on the MRT (Supplemental Figure S8), $t(180)=6.63, p<.001, d=.98$, men $(M=11.88, S D=4.12)$, women $(M=7.73, S D=4.31)$, and the SOT (Supplemental Figure $S 9)$, $t(158.02)=6.50, p<.001, d=.94$, men $(M=24.75, S D=17.57)$, women $(M=47.78, S D=$ 29.32), reported a greater sense of direction on the SBSOD (Supplemental Figure S10), $t(180)=$ 
$3.39, p=.001, d=.51$, men $(M=4.41, S D=.89)$, women $(M=3.91, S D=1.01)$, reported greater small-scale spatial ability on the PSAS (Supplemental Figure S11), $t(180)=2.63, p=$ .009 , men $(M=4.70, S D=.84)$, women $(M=4.39, S D=.74)$, and reported a greater number of video game hours per week (Supplemental Figure S12), $t(180)=4.99, p<.001, d=.74$, men $(M$ $=18.42, S D=13.12)$, women $(M=9.24, S D=11.68)$. In summary, men tended to perform better on performance-based measures of spatial ability and reported a greater sense of direction and small-scale spatial ability, as well as more video game hours.

\section{Discussion}

The present study investigated individual differences in a triangle completion task in VR using three locomotion interfaces and two VEs. The locomotion interfaces differed in available self-motion cues, and the VEs differed in available piloting cues. As predicted, absolute distance errors when pointing to the path origin were greatest for discordant teleporting, followed by partially concordant teleporting, followed by walking. Additionally, errors were consistently lower in the classroom VE compared to the open field VE. Contrary to predictions, errors were also significantly lower for walking in the classroom VE compared to walking in the open field VE. This is likely due to the larger sample size in this study $(N=182)$ compared to the sample size in Cherep, Lim, Kelly, Acharya, et al. (2020) $(N=24){ }^{2}$

Correlations among the spatial ability measures were consistent with previous literature, as the MRT, the SBSOD, the SOT, and the PSAS were all significantly associated in the expected directions. Weekly video game hours were significantly associated with the MRT and SOT, but not the SBSOD or the PSAS. This relationship follows from other work that has shown

\footnotetext{
${ }^{2}$ The magnitude of this difference (Cohen's d) was similar in both studies $(d=.23$ for the current study and $d=.20$ for Cherep et al., 2020).
} 
that computer experience (which includes video gameplay) has a positive relationship with MRT (Terlecki \& Newcombe, 2005) and SOT performance (Ventura et al., 2013).

On average, men and women performed similarly when walking, but men outperformed women with the two teleporting interfaces. This suggests that women may be more reliant on self-motion cues compared to men, who were better able to compensate for limited access to self-motion cues. This finding parallels other work on spatial updating showing that men and women perform similarly when provided with ample piloting cues but that men outperform women when piloting cues are reduced or degraded (Kelly et al., 2009). Additionally, women performed worse than men on the MRT and the SOT, reported lower spatial ability on the SBSOD and the PSAS, and reported fewer video game hours per week. It is possible that the male advantage when teleporting occurred because men, when faced with the challenge of reduced-cue locomotion, were better able to draw on the spatial cognitive resources that underlie processes such as mental rotation and perspective-taking to compensate for the lack of sensory cues.

The finding that gender differences only emerged under conditions of reduced selfmotion cues (i.e., when teleporting) should give navigation researchers pause. Navigation in the real world typically involves numerous self-motion cues, whereas navigation in VR commonly limits available self-motion cues due to reliance on locomotion interfaces. Immersive VR (e.g., VR experienced using a headset) often includes body-based and visual self-motion cues about rotation but eliminates or reduces self-motion cues associated with translation. Desktop VR never includes body-based self-motion cues and relies entirely on visual cues. Therefore, navigation research using VR might overestimate the occurrence and magnitude of gender differences compared to research conducted in the real world. 
An exploratory LPA using triangle completion errors from six conditions identified three profiles: Accurate Integrators, Inaccurate Integrators, and Inaccurate Non-Integrators. Accurate Integrators were characterized by overall accurate triangle completion performance and superior performance in the classroom VE compared to the open field VE, reflecting the fact that they integrated self-motion cues and piloting cues when available. They also exhibited good performance on the MRT and SOT, and self-reported high spatial ability on the SBSOD and PSAS. Furthermore, Accurate Integrators played more hours of video games and were predominantly male.

Triangle completion performance by Inaccurate Integrators and Inaccurate NonIntegrators was indistinguishable in the open field $\mathrm{VE}$, but their performance diverged in the classroom VE, where Inaccurate Integrators outperformed Inaccurate Non-Integrators when using the two teleporting interfaces. This divergence indicates that Inaccurate Integrators were better able to integrate self-motion cues with piloting cues in the classroom VE compared to Inaccurate Non-Integrators. It is worth noting that Inaccurate Non-Integrators did benefit from the piloting cues available in the classroom VE, suggesting that they did integrate self-motion cues with piloting cues, but they did so only modestly compared to the other two profiles. Beyond these differences in triangle completion performance, Inaccurate Integrators and Inaccurate Non-Integrators significantly differed on the SOT and differed marginally on the MRT, with Inaccurate Integrators outperforming Inaccurate Non-Integrators on both tasks. Both groups were predominantly female.

These results from the LPA and subsequent analyses are consistent with the notion that better performance on spatial measures would be related to triangle completion performance; however, self-report measures of spatial ability were less diagnostic of spatial updating 
performance when teleporting except in extreme comparisons (e.g., Accurate Integrators and Inaccurate Non-Integrators). In other words, self-report measures of spatial ability, such as the SBSOD and the PSAS appear to do well at parsing participants who perform well on all the interfaces from participants who perform poorly but do not distinguish the mid-performing profile (Inaccurate Integrators).

Compared to the performance-based spatial ability measures (MRT and SOT), self-report measures of spatial ability (SBSOD and PSAS) produced weaker correlations with triangle completion performance and only distinguished the highest-performing profile (Accurate Integrators) from the lowest-performing profile (Inaccurate Non-Integrators). Only the SOT clearly distinguished between all three profiles ${ }^{3}$. Why would the SOT uniquely differentiate performance between the three profiles? The SOT involves perspective-taking, which is assumed to involve a process whereby participants reorient themselves to the environment from novel perspectives. Likewise, triangle completion requires updating of self-orientation along the outbound path.

The three profiles identified in the LPA are interpreted as representing differences in overall accuracy as well as differences in the integration of self-motion cues and piloting cues. Differences in accuracy are evident directly in the error data, but differences in cue integration are inferred and more work is needed to determine whether those differences are due to suboptimal integration or lower accuracy when using piloting cues. Research on cue integration typically evaluates navigation performance with multiple cues and also with individual cues in isolation. Evidence for integration is found when performance with multiple cues exceeds that of

\footnotetext{
${ }^{3}$ The MRT also distinguished between all three groups, but due to the exploratory nature of the LPA we are cautious when interpreting the marginally significant performance difference between Inaccurate Integrators and Inaccurate Non-Integrators.
} 
single-cue conditions, and evidence for optimal integration is determined by whether response variability with multiple cues is reduced to optimal levels determined by a mathematical model based on single-cue response variability (Chen et al., 2017; Nardini et al., 2008; Sjolund et al., 2018; Zhang et al., 2019). To evaluate integration in this way, the current study would need to be modified by including a condition in which only piloting cues are available when returning to a learned location.

In one of the very few studies to describe individual differences in spatial updating, Hegarty et al. (2002) reported that SBSOD was significantly correlated with performance on a task in which blindfolded participants pointed to home after walking outbound paths ranging from 2-5 path segments. In the walking conditions reported here, SBSOD did not correlate with pointing performance, nor did any other measure collected outside of the VE. Individual differences in the current study only emerged when self-motion cues were removed (i.e., in the teleporting conditions). It is, therefore, possible that the individual differences in spatial updating only occur under conditions of reduced self-motion cues, such as teleporting or wearing a blindfold, or in walking conditions that involve more complex outbound paths as in Hegarty et al. (2002). Further work is needed to investigate possible boundary conditions of individual differences in spatial updating when walking.

There is a clear need to reduce disorientation in VEs, especially among those in the Inaccurate Non-Integrators profile. The current project likely underestimates the extent of disorientation in a typical VR experience, which involves much more complex exploration paths than the two-legged paths used here. Additionally, self-report data shows that $20 \%$ of users who have previously used VR belong to the Inaccurate Non-Integrators profile, which suggests that lower spatial ability and a greater propensity for disorientation do not dissuade these users from 
experiencing VR. Future research should consider whether feedback-based training with teleporting interfaces could reduce disorientation, particularly among those most susceptible. Likewise, investigation of the characteristics of piloting cues (e.g., cue quantity or cue salience) could reveal methods for creating VEs that allow all individuals to integrate self-motion and piloting cues. Furthermore, the teleporting interfaces themselves could be modified to reduce their disorienting effects. For example, the presence of another person in a scene can cause spontaneous perspective-taking (Tversky \& Hard, 2009), and the inclusion of an avatar at the selected location when teleporting might aid the to-be-taken perspective. Other modifications could include previews of the to-be-taken perspective or overhead (bird's eye view) maps showing current and selected locations. Also, adding visual cues corresponding to distance traveled during teleportation could enhance self-motion cues, though those visual cues would likely need to be crafted to not induce cybersickness via techniques such as tunneling (foveal optic flow with blurred periphery) (Lin et al., 2020; Norouzi et al., 2018).

This study was not designed to identify whether individual characteristics, such as video game hours or spatial ability, are causally related to triangle completion performance. However, the identification of such causal connections could provide training opportunities to reduce disorientation in VR. Spatial abilities are known to be malleable (Uttal et al., 2013), and if spatial abilities such as mental rotation or perspective-taking were causally related to navigation in VR, then training those skills could make VR applications more effective and more enjoyable.

The SOT was an objective measure of perspective-taking that distinguished spatial updating performance between all three profiles. This result suggests that the SOT could be administered (perhaps in a shortened form) as a predictive measure and used to personalize and adapt the interface and VE to better suit the user. For example, if an individual performs well on 
the SOT, then the VR application could suggest partially concordant or discordant teleporting interfaces alongside VEs that vary in visual piloting cues. However, if an individual scores poorly on the SOT, this suggests that the user would likely benefit from using a partially concordant interface or walking (when feasible) and VEs that include numerous visual piloting cues.

Disorientation will impede the effectiveness of VR for training and education, as well as the popularity of VR for entertainment. The results reported here are consistent with previous research showing that teleporting disrupts spatial updating, leading to disorientation (Cherep, Lim, Kelly, Acharya, et al., 2020; Kelly et al., 2020). Additionally, this work demonstrates that individuals vary in the extent to which disorientation will occur. These individual differences reflect differences in overall navigational accuracy as well as differences in cue integration. Furthermore, individual susceptibility to disorientation is related to performance on measures of spatial ability. 


\section{References}

Allen, M., Poggiali, D., Whitaker, K., Rhys Marshall, T. \& Kievit, R. A. (2019). Raincloud plots: A multi-platform tool for robust data visualization [version 1; peer review: 2 approved]. Welcome Open Res, 4(63), 1-41. https://doi.org/10.12688/wellcomeopenres.15191.1

Berlin, K. S., Williams, N. A., \& Parra, G. R. (2014). An introduction to latent variable mixture modeling (part 1): Overview and cross-sectional latent class and latent profile analyses. Journal of Pediatric Psychology, 39(2), 174-187. https://doi.org/10.1093/jpepsy/jst084

Boletsis, C. (2017). The New Era of Virtual Reality Locomotion: A Systematic Literature Review of Techniques and a Proposed Typology. Multimodal Technologies and Interaction, 1(4), 24. https://doi.org/10.3390/mti1040024

Bozgeyikli, E., Raij, A., Katkoori, S., \& Dubey, R. (2016). Point \& teleport locomotion technique for virtual reality. In A. Cox \& Z. O. Toups (Eds.), Proceedings of the 2016 Annual Symposium on Computer-Human Interaction in Play (pp. 205-216). New York, NY: Association for Computing Machinery. https://doi.org/10.1145/2967934.2968105

Chen, X., McNamara, T. P., Kelly, J. W., \& Wolbers, T. (2017). Cue combination in human spatial navigation. Cognitive Psychology, 95, 105-144. https://doi.org/10.1016/j.cogpsych.2017.04.003

Cherep, L. A., Lim, A. F., Kelly, J. W., Acharya, D., Velasco, A., Bustamante, E., Ostrander, A. G., Gilbert, S. B. (2020). Spatial cognitive implications of teleporting through virtual 
environments. Journal of Experimental Psychology: Applied, 26(3), 480-492. https://doi.org/10.1037/xap0000263

Cherep, L. A., Lim, A. F., Kelly, J. W., Miller, A., \& Gilbert, S. B. (2020). Individual differences in teleporting through virtual environments: A latent profile analysis. In Proceedings of IEEE Virtual Reality Annual Meeting (IEEE VR '20), March 22-26, 2020, Virtual. IEEE VR, Atlanta, GA. https://doi.org/10.1109/VRW50115.2020.00213.

Davies, C., Athersuch, L., \& Amos, N. (2017). Sense of direction: one or two dimensions? In 13th International Conference on Spatial Information Theory (COSIT 2017). Schloss Dagstuhl-Leibniz-Zentrum fuer Informatik. https://doi.org/10.4230/LIPIcs.COSIT.2017.9

Feng, J., Spence, I., \& Pratt, J. (2007). Playing an action video game reduces gender differences in spatial cognition. Psychological Science, 18(10), 850-855. https://doi.org/10.1111/j.1467-9280.2007.01990.x

Ferguson, A. M., Maloney, E. A., Fugelsang, J., \& Risko, E. F. (2015). On the relation between math and spatial ability: The case of math anxiety. Learning and Individual Differences, 39, 1-12. https://doi.org/10.1016/j.lindif.2015.02.007

Friedman, A., Kohler, B., Gunalp, P., Boone, A. P., \& Hegarty, M. (2019). A computerized spatial orientation test. Behavior Research Methods, 1-14. https://doi.org/10.3758/s13428-019-01277-3

Gabriel, A. S., Daniels, M. A., Diefendorff, J. M., \& Greguras, G. J. (2015). Emotional labor actors: A latent profile analysis of emotional labor strategies. Journal of Applied Psychology, 100(3), 863-879. https://doi.org/10.1037/a0037408 
Hegarty M., Crookes R.D., Dara-Abrams D., Shipley T.F. (2010) Do All Science Disciplines Rely on Spatial Abilities? Preliminary Evidence from Self-report Questionnaires. In Hölscher C., Shipley T.F., Olivetti Belardinelli M., Bateman J.A., Newcombe N.S. (Eds) Spatial Cognition VII. Spatial Cognition 2010. Lecture Notes in Computer Science, 6222. Springer, Berlin, Heidelberg. https://doi.org/10.1007/978-3-64214749-4_10

Hegarty, M., Montello, D. R., Richardson, A. E., Ishikawa, T., \& Lovelace, K. (2006). Spatial abilities at different scales: Individual differences in aptitude-test performance and spatial-layout learning. Intelligence, 34(2), 151-176.

https://doi.org/10.1016/j.intell.2005.09.005

Hegarty, M., Richardson, A. E., Montello, D. R., Lovelace, K., \& Subbiah, I. (2002).

Development of a self-report measure of environmental spatial ability. Intelligence, 30(5), 425-447. https://doi.org/10.1016/S0160-2896(02)00116-2

Hegarty, M., \& Waller, D. A. (2004). A dissociation between mental rotation and perspectivetaking spatial abilities. Intelligence, 32(2), 175-191. https://doi.org/10.1016/j.intell.2003.12.001

Hirschi, A., \& Valero, D. (2017). Chance events and career decidedness: Latent profiles in relation to work motivation. The Career Development Quarterly, 65(1), 2-15. https://doi.org/10.1002/cdq.12076

Kelly, J. W., McNamara, T. P., Bodenheimer, B., Carr, T. H. \& Rieser, J. J. (2009). Individual differences in using geometric and featural cues to maintain spatial orientation: Cue quantity and cue ambiguity are more important than cue type. Psychonomic Bulletin \& Review, 16(1), 176-181. https://doi.org/10.3758/PBR.16.1.176 
Kelly, J. W., Ostrander, A. G., Lim, A. F., Cherep, L. A., \& Gilbert, S. B. (2020). Teleporting through virtual environments: Effects of path scale and environmental scale on spatial updating. IEEE Transactions on Visualization and Computer Graphics, 26(5), 18411850. https://doi.org/10.1109/TVCG.2020.2973051

Klatzky, R. L., Loomis, J. M., Beall, A. C., Chance, S. S., \& Golledge, R. G. (1998). Spatial updating of self-position and orientation during real, imagined, and virtual locomotion. Psychological Science, 9(4), 293-298. https://doi.org/10.1111\%2F14679280.00058

Kozhevnikov, M. \& Hegarty, M. (2001). A dissociation between object manipulation spatial ability and spatial orientation ability. Memory \& Cognition, 29(5), 745-756. https://doi.org/10.3758/BF03200477

Langbehn, E., Lubos, P., \& Steinicke, F. (2018). Evaluation of locomotion techniques for roomscale VR: Joystick, teleportation, and redirected walking. In S. Richir (Ed.), Proceedings of the Virtual Reality International Conference-Laval Virtual (pp. 1-9). New York, NY: Association for Computing Machinery. https://doi.org/10.1145/3234253.3234291

Lhuillier, S., Gyselinck, V., Piolino, P., \& Nicolas, S. (2020). "Walk this way": Specific contributions of active walking to the encoding of metric properties during spatial learning. Psychological Research, 1-16. https://doi.org/10.1007/s00426-020-01415-z

Lin, Y. X., Babu, S. V., Venkatakrishnan, R., Venkatakrishnan, R., Wang, Y. C., \& Lin, W. C. (2020). Towards an Immersive Virtual Simulation for Studying Cybersickness during Spatial Knowledge Acquisition. In 2020 IEEE Conference on Virtual Reality and 3D User Interfaces Abstracts and Workshops (VRW) (pp. 625-626). IEEE. https://doi.org/10.1109/VRW50115.2020.00163 
Lubke, G., \& Neale, M. C. (2006). Distinguishing between latent classes and continuous factors: Resolution by maximum likelihood?. Multivariate Behavioral Research, 41(4), 499-532. https://doi.org/10.1207/s15327906mbr4104_4

Muthén, L. K. \& Muthén, B. O. (1998-2012). Mplus User’s Guide. Seventh Edition. Los Angeles, CA: Muthén \& Muthén.

Nardini, M., Jones, P., Bedford, R., \& Braddick, O. (2008). Development of cue integration in human navigation. Current Biology, 18(9), 689-693. https://doi.org/10.1016/j.cub.2008.04.021

Norouzi, N., Bruder, G., \& Welch, G. (2018). Assessing vignetting as a means to reduce VR sickness during amplified head rotations. In Proceedings of the 15th ACM Symposium on Applied Perception (pp. 1-8). Vancouver, BC, Canada: Association for Computing Machinery. https://doi.org/10.1145/3225153.3225162

Oberski, D. (2016). Mixture models: Latent profile and latent class analysis. In J. Robertson \& M. Kaptein (Eds.) Modern statistical methods for HCI (pp. 275-287). Springer International Publishing. https://doi.org/10.1007/978-3-319-26633-6_12

Quaiser-Pohl, C., Geiser, C., \& Lehmann, W. (2006). The relationship between computer-game preference, gender, and mental-rotation ability. Personality and Individual Differences, 40(3), 609-619. https://doi.org/10.1016/j.paid.2005.07.015

Rahimi Moghadam, K., Banigan, C., \& Ragan, E. D. (2018). Scene Transitions and Teleportation in Virtual Reality and the Implications for Spatial Awareness and Sickness. IEEE Transactions on Visualization and Computer Graphics, 1, 115. https://doi.org/10.1109/tvcg.2018.2884468 
Ruddle, R. A., \& Lessels, S. (2006). For efficient navigational search, humans require full physical movement, but not a rich visual scene. Psychological Science, 17(6), 460-465. https://doi.org/10.1111\%2Fj.1467-9280.2006.01728.x

Sholl, M. J., Kenny, R. J., \& DellaPorta, K. A. (2006). Allocentric-heading recall and its relation to self-reported sense-of-direction. Journal of Experimental Psychology: Learning, Memory, and Cognition, 32(3), 516-533. https://psycnet.apa.org/doi/10.1037/02787393.32.3.516

Sjolund, L. A., Kelly, J. W., \& McNamara, T. P. (2018). Optimal combination of environmental cues and path integration during navigation. Memory \& Cognition, 46(1), 89-99. https://doi 10.3758/s13421-017-0747-7

Spence, I., \& Feng, J. (2010). Video games and spatial cognition. Review of General Psychology, 14(2), 92-104. https://doi.org/10.1037/a0019491

Spurk, D., Hirschi, A., Wang, M., Valero, D., \& Kauffeld, S. (2020). Latent profile analysis: a review and "how to" guide of its application within vocational behavior research. Journal of vocational behavior, 103445. https://doi.org/10.1016/j.jvb.2020.103445

Terlecki, M. S., \& Newcombe, N. S. (2005). How important is the digital divide? The relation of computer and videogame usage to gender differences in mental rotation ability. Sex Roles, 53(5), 433-441. https://doi.org/10.1007/s11199-005-6765-0

Tversky, B., \& Hard, B. (2009). Embodied and disembodied cognition: Spatial perspectivetaking. Cognition, 110, 124-129. https://doi.org/10.1016/j.cognition.2008.10.008

Uttal, D. H., Meadow, G., Tipton, E., Hand, L. L., Alden, A. R., Warren, C., \& Newcombe, N. S. (2013). The malleability of spatial skills: A meta-analysis of training studies. Psychological Bulletin, 139(2), 352-402. https://doi.org/10.1037/a0028446 
Vandenberg, S. G., \& Kuse, A. R. (1978). Mental rotations, a group test of three-dimensional spatial visualization. Perceptual and Motor Skills, 47(2), 599-604. https://doi.org/10.2466\%2Fpms.1978.47.2.599

Ventura, M., Shute, V., Wright, T. J., \& Zhao, W. (2013). An investigation of the validity of the virtual spatial navigation assessment. Frontiers in Psychology, 4, 852. https://doi.org/10.3389/fpsyg.2013.00852

Vermunt, J. K., \& Magidson, J. (2002). Latent class cluster analysis. In J. Haenaars \& A. McCutcheon (Vol. Eds.). Applied Latent Class Analysis (Vol. 11). Applied latent class analysis (pp. 89-106). Cambridge University Press. https://doi.org/10.1017/CBO9780511499531.004

Wang, M.-C., Deng, Q., Bi, X., Ye, H., \& Yang, W. (2017). Performance of the entropy as an index of classification accuracy in latent profile analysis: A Monte Carlo simulation study. Acta Psychologica Sinica, 49(11), 1473-1482. https://doi.org/10.3724/SP.J.1041.2017.01473

Weisberg, S. M., \& Newcombe, N. S. (2016). How do (some) people make a cognitive map? Routes, places, and working memory. Journal of Experimental Psychology: Learning, Memory, and Cognition, 42(5), 768. http://dx.doi.org/10.1037/xlm0000200

Weisberg, S. M., Schinazi, V. R., Newcombe, N. S., Shipley, T. F., \& Epstein, R. A. (2014). Variations in cognitive maps: Understanding individual differences in navigation. Journal of Experimental Psychology: Learning, Memory, and Cognition, 40(3), 669-682. https://doi.org/10.1037/a0035261

Woo, S. E., Jebb, A. T., Tay, L., \& Parrigon, S. (2018). Putting the "person" in the center: Review and synthesis of person-centered approaches and methods in organizational 
science. Organizational Research Methods, 21(4), 814-845.

https://doi.org/10.1177\%2F1094428117752467

Zhang, L. \& Mou, W. (2017). Piloting systems reset path integration systems during position estimation. Journal of Experimental Psychology: Learning, Memory, and Cognition, 43(3), 472-491. https://doi.org/10.1037/xlm0000324

Zhang, L., Mou, W., Lei, X., \& Du, Y. (2019). Cue combination used to update the navigator's self-localization, not the home location. Journal of Experimental Psychology: Learning, Memory, and Cognition. https://doi.org/10.1037/xlm0000794

Zhao, M., \& Warren, W. H. (2015). How you get there from here: Interaction of visual landmarks and path integration in human navigation. Psychological Science, 26(6), 915924. https://doi.org/10.1177\%2F0956797615574952 